Check for updates

Cite this: RSC Adv., 2018, 8, 10806

Received 2nd February 2018

Accepted 22nd February 2018

DOI: $10.1039 / c 8 r a 01059 e$

rsc.li/rsc-advances

\section{A conductive sodium alginate and carboxymethyl chitosan hydrogel doped with polypyrrole for peripheral nerve regeneration}

\author{
Ying $\mathrm{Bu}^{\mathrm{a}}{ }^{\mathrm{H}} \mathrm{Hai}-\mathrm{Xing} \mathrm{Xu},{ }^{\text {*a }}$ Xin $\mathrm{Li}{ }^{\mathrm{a}}$ Wen-Jin $\mathrm{Xu},{ }^{\mathrm{a}}$ Yi-xia Yin, ${ }^{\mathrm{b}}$ Hong-lian Dai, ${ }^{\mathrm{b}}$ \\ Xiao-bin Wang, ${ }^{c}$ Zhi-Jun Huang ${ }^{a}$ and Pei-Hu Xu*a
}

\begin{abstract}
Polymer materials with electrically conductive properties have good applications in their respective fields because of their special properties. However, they usually exhibited poor mechanical properties and biocompatibility. In this work, we present a simple approach to prepare conductive sodium alginate (SA) and carboxymethyl chitosan (CMCS) polymer hydrogels (SA/CMCS/PPy) that can provide sufficient help for peripheral nerve regeneration. SA/CMCS hydrogel was cross-linked by calcium ions provided by the sustained release system consisting of D-glucono- $\delta$-lactone (GDL) and superfine calcium carbonate $\left(\mathrm{CaCO}_{3}\right)$, and the conductivity of the hydrogel was provided by doped with polypyrrole (PPy). Gelation time, swelling ratio, porosity and Young's modulus of the conductive SA/CMCS/PPy hydrogel were adjusted by polypyrrole content, and the conductivity of it was within $2.41 \times 10^{-5}$ to $8.03 \times$ $10^{-3} \mathrm{~S} \mathrm{~cm}^{-1}$. The advantages of conductive hydrogels in cell growth were verified by controlling electrical stimulation of cell experiments, and the hydrogels were also used as a filling material for the nerve conduit in animal experiments. The SA/CMCS/PPy conductive hydrogel showed good biocompatibility and repair features as a bioactive biomaterial, we expect this conductive hydrogel will have a good potential in the neural tissue engineering.
\end{abstract}

\section{Introduction}

Hydrogel is a soft and high plasticity polymer material. ${ }^{1}$ It can be obtained by chemical crosslinking or physical crosslinking by natural and synthetic polymers. Generally, natural polymers are widely used in biological materials because of their cheap price, simple synthesis and high biocompatibility. When the hydrogels are synthesized from polymer materials, different functions are given due to the properties of the materials themself or the needs of the experiments. Some environmentally sensitive hydrogels were used as sensors or controlledrelease switches, because the properties of hydrogels changed correspondingly after the change of temperature, $\mathrm{pH}$ or pressure. For example, chitosan (CS)/polyvinyl alcohol (PVA) hydrogels regulate $\mathrm{pH}$ to control the drug release. ${ }^{2}$ Some hydrogels were used as tissue engineering materials to improve the mechanical properties, such as the composite hydrogel made of cellulose nanocrystals skeleton and polyacrylamide, its

\footnotetext{
${ }^{a}$ Department of Pharmaceutical Engineering, School of Chemistry, Chemical Engineering and Life Sciences, Wuhan University of Technology, Wuhan 430070, PR China

${ }^{b}$ Biomedical Materials and Engineering Research Center, Wuhan University of Technology, Wuhan 430070, PR China

${ }^{c}$ Wuhan Kanghua Century Pharmaceutical Company, Wuhan 430070, PR China. E-mail: 3186@whut.edu.cn; whutxph68@126.com; Tel: +862787749300
}

elasticity and hardness have been greatly improved, which can be used as a scaffold for tissue engineering. ${ }^{3,4}$ Natural polymer hydrogel has excellent biocompatibility, large surface area and porous structure, its extracellular matrix (ECM)-mimicked structure can be well applied to cell adhesion and proliferation. ${ }^{5,6}$ Natural polymers have been designed as materials with different functions due to their differences in properties, many studies have reported that functional biomaterials based on chitosan (CS) or sodium alginate (SA). ${ }^{7}$ In the existing results, we found that the synthesized materials are based on CMCS and SA have the characteristics of biocompatibility and biodegradability of high biomass. ${ }^{8,9}$ Combined with the analysis and research of synthetic hydrogels, we know that their structure is porous network structure, and we can adjust the structure and mechanical properties of hydrogels by changing the synthetic reaction conditions and composition materials. ${ }^{\mathbf{1 0 , 1 1}}$ This kind of hydrogels are based on natural polymer materials and can be used as cell culture, loading drugs and supporting materials for tissue regeneration. ${ }^{\mathbf{1 2 , 1 3}}$ Once the functional hydrogels were prepared, they may play a role in replacing the defect tissue and treating the human injuries.

Among a large number of human injuries, nerve damage has been relatively difficult to cure, which allows us to make a lot of research in nerve repair work. There are many types of nerve injuries, and are usually accompanied by trauma around the tissues. ${ }^{14-16}$ Therefore, a material that acts on the injured site to 
promote nerve repair and wound healing around needs to be found. In the existing solutions, we have focused on the use of nerve conduits to repair the nerve injury. At present, some tissue engineering materials with high biocompatibility and high mechanical strength have been applied to segmental nerve repair. For example, functional neural conduit is applied to long segment nerve injury such as sciatic nerve injury and achieved good results. ${ }^{17-19}$ Through a series of studies, it is not difficult to find that during the process of nerve repair, we need to provide a good environment for the growth, proliferation and differentiation of nerve cells, including no negative stimuli, certain nutrients, growth space, and easy to stick to migration. Therefore, we want to make full use of the characteristics of natural polymer hydrogels, such as good biocompatibility and porous structure, to serve as a basic carrier for placement of nerve damage. At the same time, we also learned that appropriate electrical stimulation can induce nerve cell differentiation and promote axonal growth of neurons. ${ }^{20-22}$ However, generally the biodegradability of materials with conductivity can not satisfy all expectations. We hope to combine the biocompatible polymer hydrogels with conductive materials, and create a functional conductive hydrogel that can provide strong help for nerve repair and tissue regeneration. Therefore, in the field of nerve repair, all researches need to be restored to the best direction and minimize negative effects. ${ }^{23,24}$

In this study, a SA/CMCS/PPy conductive hydrogel was fabricated. SA and CMCS were crosslinked by $\mathrm{Ca}^{2+}$, and PPy was doped in the ECM-mimicked SA/CMCS structure. ${ }^{25,26}$ Different from the traditional single hydrogels, the conductive hydrogel was given controllable conductivity and proper mechanical properties. The hydrogel was evaluated by cultivating PC12, RSC96, BMMSC cells and completing animal experiments as a filling material of nerve conduit. ${ }^{27-29}$ The aim of this work is to explore the performance of SA/CMCS/PPy hydrogel and to prove the potential application of the conductive hydrogel in the neural tissue engineering.

\section{Materials and methods}

\subsection{Materials}

Sodium alginate (SA), carboxymethyl chitosan (CMCS) and pyrrole (Py) were purchased from Sinopharm Chemical Reagent Ltd (China). Py was distilled under reduced pressure and stored at $3{ }^{\circ} \mathrm{C}$ in a dark area. $\mathrm{D}$-Glucono- $\delta$-lactone (GDL) was purchased from Shanghai Aladdin Biochemical Ltd (Shanghai, China). Superfine calcium carbonate $\left(\mathrm{CaCO}_{3}\right)$ was purchased from Hejian Zijin Ltd (Hejian, China). Ammonium persulfate $\left(\left(\mathrm{NH}_{4}\right)_{2} \mathrm{~S}_{2} \mathrm{O}_{8}\right)$ was purchased from Sinopharm Chemical Reagent Ltd (China). Poly-DL-lactide (PDLLA) was purchased from Jinan Daigang Bioengin Ltd (Wuhan, China). DiIC18(3) (Dil) and 6diamidino-2-phenylindole (DAPI) were purchased from Biyuntian Ltd (Wuhan, China). All other reagents and solvents used in the study were of the highest analytical purity and were purchased from Wuhan Meisitong Biochem Ltd (Wuhan, China).

\subsection{Synthesis of SA/CMCS hydrogel}

SA/CMCS hydrogel was synthesized from SA and CMCS with a series of weight ratios from $3: 1$ to $1: 3 . \mathrm{Ca}^{2+}$ was used as a crosslink reagent and the amount was equivalent to the reaction with the carboxyl. For example, SA (3 g) and CMCS (3 g) were weighted respectively in a round bottom flask, the $100 \mathrm{~mL}$ deionized water was added to the solution, and the concentration of $0.03 \mathrm{~g} \mathrm{~mL}^{-1}$ was obtained after the full swelling. $\mathrm{Ca}^{2+}$ was provided by the system of GDL and superfine $\mathrm{CaCO}_{3}$, and the ratio of the amount of GDL to superfine $\mathrm{CaCO}_{3}$ is $2: 1$. The mixture of SA and CMCS solution was quickly mixed at $37^{\circ} \mathrm{C}$, and superfine $\mathrm{CaCO}_{3}$ powder and GDL were added in order. After settling for a period of time, the homogeneous gel product was obtained.

\subsection{Synthesis of PPy}

Preparation of hydrochloric acid (HCL) solution for $1.0 \mathrm{~mol} \mathrm{~L}^{-1}$. Added $1.4 \mathrm{~mL}$ pyrrole (Py) to $75 \mathrm{~mL}$ HCL solution, ultrasonic agitation, then it was placed in a cryogenic reactor at $0{ }^{\circ} \mathrm{C}$ for 30 minutes. Removed ammonium persulfate $2.74 \mathrm{~g}$ dissolved in $25 \mathrm{~mL}$ deionized water. After completely dissolving, it was also preserved for 30 minutes at $0{ }^{\circ} \mathrm{C}$. The HCL solution of ammonium persulfate was dripped into a HCL solution containing Py at a rate of $3 \mathrm{~mL} \min ^{-1}$ without stirring, and kept the reaction $24 \mathrm{~h}$ at $0{ }^{\circ} \mathrm{C}$. At the end of the reaction, the product with a large amount of distilled water washing and filtration, vacuum drying at $60{ }^{\circ} \mathrm{C}$ for $48 \mathrm{~h}$ until constant weight, and finally got the black PPy powder.

\subsection{Preparation of SA/CMCS/PPy hydrogel}

The SA and CMCS solution with a concentration of $0.03 \mathrm{~g} \mathrm{~mL}^{-1}$ were prepared respectively, and then the $20 \mathrm{~mL}$ SA solution was mixed with the $10 \mathrm{~mL}$ CMCS solution and stirred evenly. The PPy powder produced by the above method was weighed and then added to SA/CMCS solution at 0.02, 0.05, 0.10, 0.20 and 0.40 according to the mass ratio of the feed, stirred evenly after ultrasonic vibration. The nano- $\mathrm{CaCO}_{3}$ powder of $0.113 \mathrm{~g}$ and GDL of $0.407 \mathrm{~g}$ were added step by step, and SA/CMCS/PPy hydrogel was obtained after a period of quiet time.

\subsection{Characterization of the hydrogels}

FTIR spectra of SA, CMCS, Py, PPy, CMCS/SA hydrogel, and CMCS/SA/PPy hydrogel were recorded on a FT-IR spectrometer (Nicolet 6700, Thermo Electron Scientific Instrument, USA) in the range of $4000-600 \mathrm{~cm}^{-1}$.

The sol-gel transition time at body temperature was determined by tube inverting test. The sample was defined as a "gel" in the case of no visual flow was observed within $30 \mathrm{~s}$ by inverting the vial.

The swelling ratio (SR) of the hydrogels was determined by swelling tests. The hydrogels were immersed in PBS solution and weighed from the solution without external water at various times $\left(W_{t}\right)$. Hydrogels were weighed until swelling equilibrium was established. SR was calculated by the following equation: 
$\mathrm{SR}=\left(W_{t} / W_{i}\right) \%$, where $W_{i}$ and $W_{t}$ represent the initial weight and the weight at different swelling times, respectively.

The porosity was measured by the specific gravity method. The freeze-dried hydrogel was cut into a regular shape of a certain volume, and the volume of the gel was calculated to be $V_{\mathrm{e}}$, and the weight of the gel was recorded by $W_{0}$. The hydrogel was placed in a sealed container filled with ethanol, and the weight of the sample was recorded as $W_{\mathrm{E}}$ after the ethanol was completely filled in the pore of the hydrogel. The weight of the liquid in the pores of the hydrogel was equal to the weight difference between the $W_{\mathrm{E}}$ and $W_{0}$, according to the density of liquid ethanol could calculate the pore volume $V_{\mathrm{P}}=\left(W_{\mathrm{E}}-W_{0}\right) / \rho$ and porosity $P(\%)=\left(V_{\mathrm{P}} / V_{\mathrm{e}}\right) \%$.

Cyclic voltammetry (CV) of CMCS/SA/PPy hydrogel was conducted on a CHI 660D Electrochemical Workstation using saturated calomel and platinum-wire as the reference and counter electrodes respectively. The indium tin oxide (ITO) electrode was used as the working electrode and the scan rate was $50 \mathrm{mV} \mathrm{s}^{-1}$. The conductivity of the CMCS/SA/PPy hydrogel was tested by the 4-point probe method using the Hall Effect testing system (HL5500PC, Accent Optical, UK).

The mechanical properties of the hydrogels were tested using a table universal testing machine (SHT4106, Mester Industrial System, China Ltd.). The hydrogels were cut into a cube with a border length of $12 \mathrm{~mm}$ for the axial testing with a cross-head speed of $5 \mathrm{~mm} \mathrm{~min}^{-1}$. The mechanical sensor used the specifications of the $10 \mathrm{~N}$. Tensile strength and Young's modulus were calculated based on the stress-strain curve of each sample.

The thermal behaviors of the hydrogels were investigated by thermogravimetric analysis (TGA) and differential scanning calorimetry (DSC). Thermal analyzer (STA449F3, Netzsch, Germany) heated hydrogel samples from room temperature to $800{ }^{\circ} \mathrm{C}\left(10{ }^{\circ} \mathrm{C} \mathrm{min}^{-1}\right)$ in a dynamic $\mathrm{N}_{2}$ atmosphere (flow rate $=$ $\left.20 \mathrm{~mL} \mathrm{~min}^{-1}\right)$.

After freeze drying, the morphologies of the hydrogels were observed using a field emission scanning electron microscope (JSM-IT300, JEOL, Japan). The morphology of the hydrogel and the morphologies under different degrees of degradation were included.

\subsection{In vitro cell study}

PC12, RSC96 and BMMSC cells were all purchased from Shanghai Institutes for Biological Sciences, China. PC12 cells were cultured in Roswell Park Memorial Institute (RPMI) supplemented with $5 \%$ horse serum, $10 \%$ fetal bovine serum and $1 \%$ antibiotic/antimycotic solution. RSC96 cells were cultured in Dulbecco's Modified Eagle Medium (DMEM) supplemented with $10 \%$ fetal bovine serum and $1 \%$ antibiotic/antimycotic solution. BMMSC cells were cultured in alpha Modified Eagle Medium ( $\alpha$-MEM) supplemented with $10 \%$ fetal bovine serum and $1 \%$ antibiotic/antimycotic solution. In cell culture, there were the following groups: SA/CMCS hydrogel, SA/CMCS hydrogel with electrical stimulation, SA/CMCS/PPy hydrogel, SA/CMCS/PPy hydrogel that was accompanied by electrical stimulation. After the hydrogels were sterilized under UV light for $3 \mathrm{~h}$, they were put in 24-well plates and washed with PBS. Then the cells were seeded on the hydrogels at a density of $2 \times$ $10^{4}$ cells per $\mathrm{cm}^{2}$. A $100 \mathrm{mV}$ potential was then applied across the wires for $2 \mathrm{~h}$ a day. The cells were incubated at $37{ }^{\circ} \mathrm{C}$ with $5 \%$ $\mathrm{CO}_{2}$ and the medium was replaced every 2 days. Sampling observation at 1, 3, 5 days, the samples were flushed with PBS and fixed with paraformaldehyde. The cells were fixed for 30 min and permeated using $0.1 \%$ Triton X-100. After washing with PBS, cells were dyed with red fluorescent dye Dil and nuclei was stained with 4,6-diamidino-2-phenylindole hydrochloride (DAPI). Samples were then imaged by confocal laser scanning microscopy (BX60, Olympus Optical, Japan Ltd.). The biocompatibility of the material also can be assessed by observing the cell growth. In the same way, the materials were dehydrated by gradient concentration of ethanol and dried in vacuum, then SEM was used to observe the adhesion of PC12 on the material.

\subsection{In vivo inflammatory response after subcutaneous injection}

Adult Sprague-Dawley (SD) rats were purchased from experimental animal research center of Hubei CDC (Wuhan, Hubei, China). All experiments were carried out in accordance with current guidelines for the care of laboratory animals and were approved by proper committee of Wuhan University of Technology. The SA/CMCS hydrogels, SA/CMCS/PPy hydrogels were sterilized under high temperature and pressure. Two groups of Sprague-Dawley (SD) rats (150 g, male) were anesthetized by isofluorane before being buried subcutaneously with hydrogels of $10 \mathrm{~mm} \times 5 \mathrm{~mm} \times 2 \mathrm{~mm}$ volume. The inflammatory response and histological examination were performed on three groups of animals. The injectable samples were retrieved at 1 week, 3 weeks and 5 weeks after surgery, processed for further histological analysis. At the pre-determined time points, the animals were sacrificed, and the hydrogels with inflamed tissue were removed, fixed in a phosphate buffered formalin solution, embedded in paraffin, sectioned for further histological examination. All species were stained by Hematoxylin-Eosin (H\&E). The stained sections of each sample were examined by a microscope for tissue inflammatory reaction evaluation.

\subsection{Animal experiment of nerve conduit filling material}

Adult Sprague Dawley (SD) rats were purchased from experimental animal research center of Hubei CDC (Wuhan, Hubei, China). All animal procedures were performed in accordance with the Guidelines for Care and Use of Laboratory Animals of Wuhan University of Technology, China and approved by the Animal Ethics Committee of Wuhan University of Technology. The SD rats were divided into 3 groups, each with 15 rats, and the weight of each rat was about $150 \mathrm{~g}$. The animals were anesthetized with $50 \mathrm{mg} \mathrm{kg}^{-1}$ body weight pentobarbital sodium. After the rats were anesthetized, the operation was performed and the sciatic nerve of the left hind limbs of each mouse was artificially removed. The length of the sciatic nerve was $10 \mathrm{~mm}$. Nerve conduit derived from PDLLA by solvent evaporation. The PDLLA nerve conduit with a length of $15 \mathrm{~mm}$ was used as a basic treatment to repair the damaged nerve, and 
at the same time, the filling materials in the nerve conduit were grouped into SA/CMCS hydrogel, SA/CMCS/PPy hydrogel and autologous nerve graft. After the operation, the muscle layer was reapproximated with $4-0$ chromic gut sutures, and the skin was closed with 2-0 silk sutures. At 1 and 2 months after operation, rats were taken for experimental materials, and muscle and nerve tissue morphology was assessed to observe nerve regeneration.

\subsection{Statistical analysis}

All numerical data were given as mean \pm standard deviation. SPSS 12.0 software (SPSS, USA) was used for statistical analysis of the relevant data with one-way analysis of variance (ANOVA). The data were presented as the mean \pm standard deviation. A value of $p<0.05$ was considered statistically significant.

\section{Results and discussion}

\subsection{Preparation of SA/CMCS/PPy hydrogel}

Pyrrole (PY) was oxidized and polymerized to PPY in a HCL solution using ammonium persulfate as an oxidant. The SA solution and CMCS solution were used to prepare the SA/CMCS hydrogel through $\mathrm{Ca}^{2+}$ that was provided by the system of GDL and superfine $\mathrm{CaCO}_{3}$, and they reacted with each other to form clathrates. The SA/CMCS/PPy hydrogel was obtained by adding PPy powder to the SA/CMCS hydrogel (Fig. 1(A)). Attenuated total reflectance Fourier transform infrared spectroscopy (ATRFTIR) was used to obtain the FTIR spectra of the PPy, SA/CMCS hydrogel, and SA/CMCS/PPy hydrogel (Fig. 1(B)). Compared Py with PPy in the spectrum, the bands at $1553 \mathrm{~cm}^{-1}, 1314 \mathrm{~cm}^{-1}$ and $1033 \mathrm{~cm}^{-1}$ are characteristic PPY peaks. In the spectrum of SA and CMCS, the bands at $1611 \mathrm{~cm}^{-1}, 1416 \mathrm{~cm}^{-1}$ are characteristic SA peaks, and the bands at $1116 \mathrm{~cm}^{-1}, 1025 \mathrm{~cm}^{-1}$ are characteristic CMCS peaks. In curve of SA/CMCS hydrogel, the peak at $1421 \mathrm{~cm}^{-1}$ is symmetry stretching vibration of $-\mathrm{COO}$, and because of the interaction between molecules, it moves to the high wave number. The peak of $\mathrm{C}-\mathrm{N}$ is enhanced at $1126 \mathrm{~cm}^{-1}$, and the peak of C-O peak is weakened at $1034 \mathrm{~cm}^{-1}$, indicating that the crosslinking reaction between SA and CMCS. All the above peaks are present in the SA/CMCS/PPy hydrogel spectrum, verifying that the SA/CMCS/PPy hydrogel was successfully synthesized. The photographic images and scanning electron micrographs of the PPY powder and freeze-dried SA/CMCS/PPy hydrogel were shown in Fig. 1(C). It showed that the PPy powders are spherical particles with similar specifications, and the PPy particles were uniformly dispersed on the network structure of the hydrogel skeleton.

\subsection{Gelation time measurement}

For hydrogels, the gelation time is an important factor for practical applications. The gelation time was investigated by tube inverting technique. ${ }^{30}$ The hydrogels were divided into two groups according to the mass ratio of CMCS : SA and the feed ratio of PPy. Through observing the appearance of the solution

A
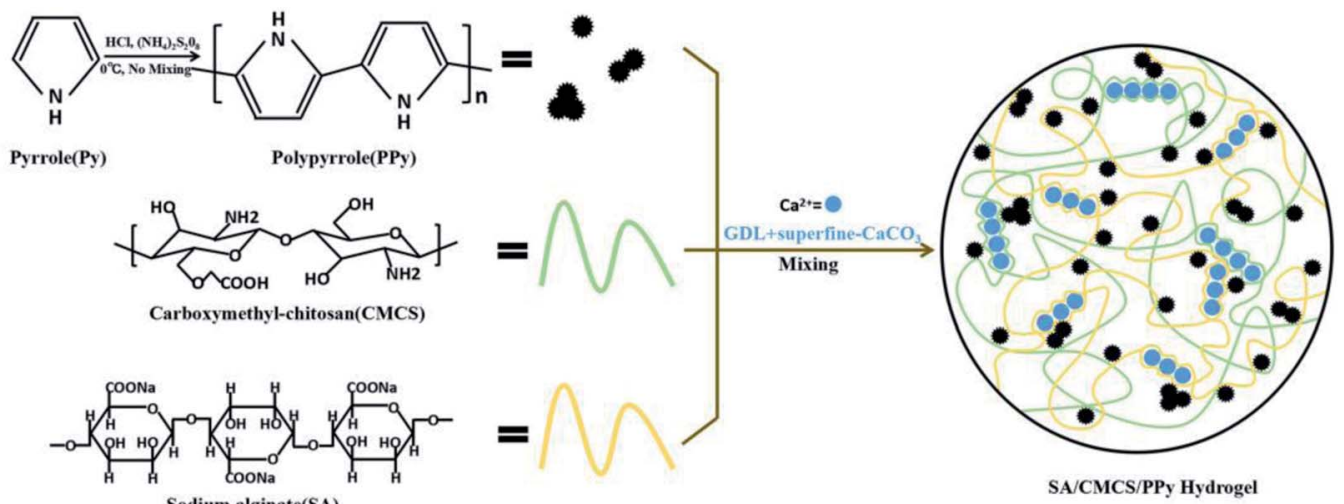

B
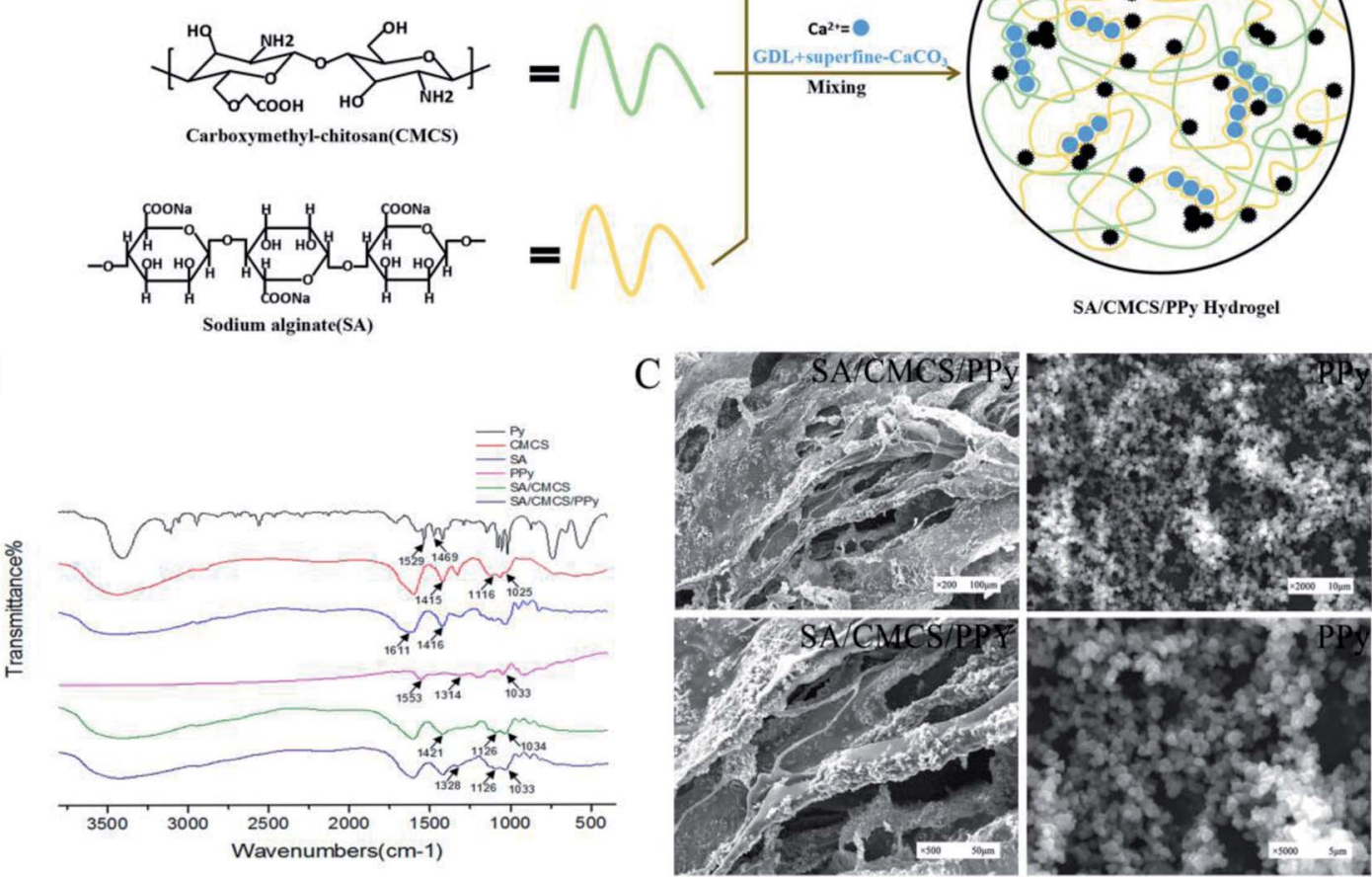

Fig. 1 (A) Preparation of PPy and electrically conductive SA/CMCS/PPy hydrogel. (B) FT-IR spectra of samples. (C) Scanning electron microscopy images of internal structures of SA/CMCS/PPy hydrogel and PPy particles. 
A

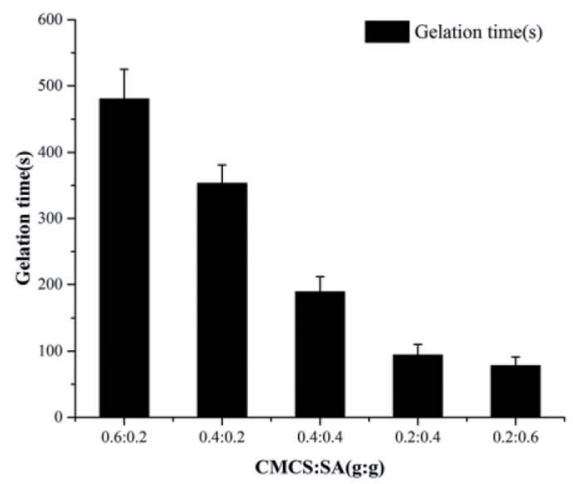

C

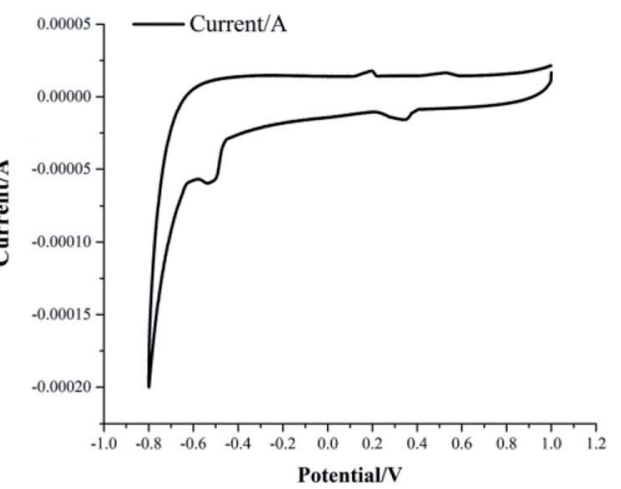

$\mathrm{B}$

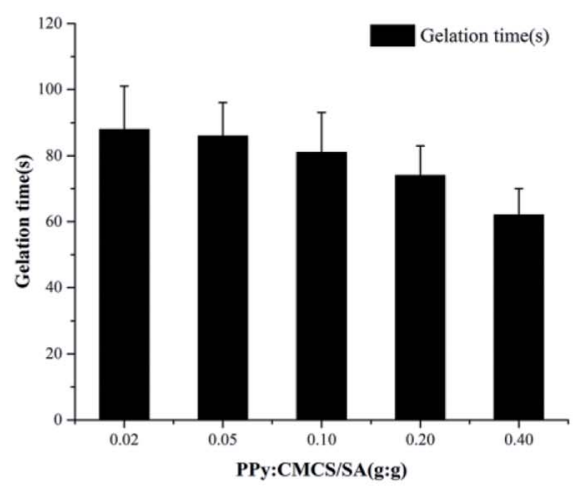

$\mathrm{D}$

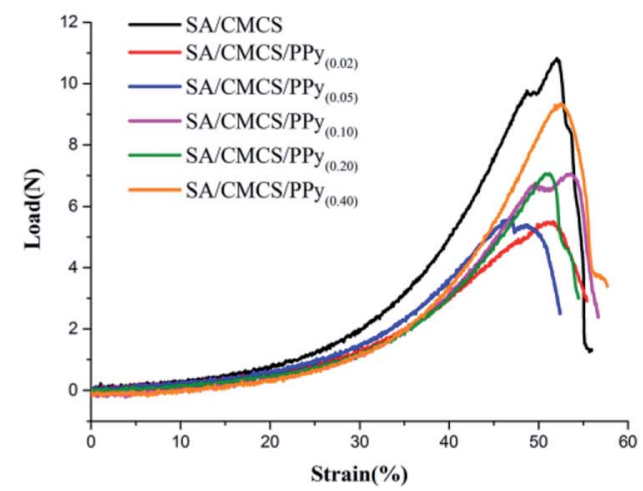

Fig. 2 (A) and (B) Gelation time of hydrogels synthesized by different conditions. (C) Cyclic voltammograms for the SA/CMCS/PPy hydrogel. (D) Compressive load-strain curves of SA/CMCS hydrogel doped with different mass ratio PPy.

and hydrogels, the SA/CMCS hydrogels were translucent pale yellow, and the SA/CMCS/PPy hydrogels were black in color. The hydrogels were formed after being placed in a $37^{\circ} \mathrm{C}$ oven for a while. The gelation times of the SA/CMCS hydrogels were between 480 seconds and 78 seconds (Fig. 2(A)), and the time was shortened with the increase of the amount of SA. As a tissue material, hydrogels should have a suitable gelation time for convenience of application, not too long or too short. ${ }^{31}$ When the mass ratio of SA: CMCS was $2: 1$, the gelation time was 94 seconds. Under this condition, the gelation times of the SA/ CMCS/PPy hydrogels were between 88 seconds and 62 seconds (Fig. 2(B)), and the gelation time continued to decrease with the increase in the amount of PPy admixture. When the feed ratio was $1: 5$, the gelation time of the SA/CMCS/PPy hydrogel was 74 seconds.

\subsection{Swelling and porosity experiments}

Based on the results of the experiments that had been done, the mass ratio of SA : CMCS $(2: 1)$ was selected as the base condition to continue the test. In order to examine the swelling stability of the hydrogels, fully swollen SA/CMCS/PPy hydrogels were dried in a vacuum freeze-drying machine, and then reimmersed in PBS solution. ${ }^{32}$ The SA/CMCS/PPy hydrogels show a high swelling ratio (SR) as indicated in Table 1 . The swelling ratio decreased as PPy content increased hence the SR can be controlled from 9.132 to 5.013 by tuning the PPy content in the hydrogels. The significant decrease of SR of the SA/CMCS/ PPy hydrogel was observed due to the hydrophobic nature of
Table 1 The characteristics of hydrogels with different PPy mass ratio

\begin{tabular}{|c|c|c|c|}
\hline Sample & $\begin{array}{l}\text { Swelling ratio } \\
(\mathrm{w} / \mathrm{w})\end{array}$ & $\begin{array}{l}\text { Porosity } \\
(\%)\end{array}$ & Conductivity $\left(\mathrm{S} \mathrm{cm}^{-1}\right)$ \\
\hline $\mathrm{SA} / \mathrm{CMCS} / \mathrm{PPy}_{(0.02)}$ & 9.132 & 86.69 & $7.35 \times 10^{-6}$ \\
\hline $\mathrm{SA} / \mathrm{CMCS} / \mathrm{PPy}_{(0.05)}$ & 9.020 & 84.27 & $2.41 \times 10^{-5}$ \\
\hline $\mathrm{SA} / \mathrm{CMCS} / \mathrm{PPy}_{(0.10)}$ & 8.461 & 80.54 & $4.71 \times 10^{-5}$ \\
\hline $\mathrm{SA} / \mathrm{CMCS} / \mathrm{PPy}_{(0.20)}$ & 7.511 & 73.16 & $1.42 \times 10^{-3}$ \\
\hline $\mathrm{SA} / \mathrm{CMCS} / \mathrm{PPy}_{(0.40)}$ & 5.013 & 62.87 & $8.03 \times 10^{-3}$ \\
\hline
\end{tabular}

PPy. When the amount of PPy was 0.20, the SR of the SA/CMCS/ PPy hydrogel was 7.511. To understand the basic condition of the pores in the SA/CMCS/PPy hydrogel, we also inspected the porosity of the hydrogels. As shown in Table 1, the range of the porosity of the SA/CMCS/PPy hydrogels was from $86.69 \%$ to $62.87 \%$. With the increase of PPy content, the porosity of hydrogels decreased. It might because many polypyrrole particles were attached to the holes in the internal structure of the hydrogels, which occupied a certain space in physics. The porosity of the SA/CMCS/PPy hydrogel was $73.16 \%$ in the condition that feeding ratio of polypyrrole was 0.20 .

\subsection{Electrochemical characterization of SA/CMCS/PPy conducting hydrogel}

Cyclic voltammetry (CV) has been widely used to investigate the electrochemical properties of electro-active polymers. ${ }^{33,34}$ Detection by three electrode system, Fig. 2(C) shows the cyclic voltammogram of the $\mathrm{SA} / \mathrm{CMCS} / \mathrm{PPy}_{(0.20)}$ hydrogel in sodium 
Table 2 The mechanical properties of hydrogels with different PPy mass ratio

\begin{tabular}{|c|c|c|}
\hline Sample & $\begin{array}{l}\text { Tensile stress } \\
(\mathrm{MPa})\end{array}$ & $\begin{array}{l}\text { Young's } \\
\text { modulus (MPa) }\end{array}$ \\
\hline SA/CMCS & 0.07516 & 0.42952 \\
\hline $\mathrm{SA} / \mathrm{CMCS} / \mathrm{PPy}{ }_{(0.02)}$ & 0.03823 & 0.17470 \\
\hline SA/CMCS/PPy $(0.05)$ & 0.03938 & 0.23199 \\
\hline $\mathrm{SA} / \mathrm{CMCS} / \mathrm{PPy}_{(0.10)}$ & 0.04898 & 0.28255 \\
\hline $\mathrm{SA} / \mathrm{CMCS} / \mathrm{PPy}_{(0.20)}$ & 0.04902 & 0.28898 \\
\hline $\mathrm{SA} / \mathrm{CMCS} / \mathrm{PPy}_{(0.40)}$ & 0.06494 & 0.40595 \\
\hline
\end{tabular}

chloride hydrochloric acid solution. In Fig. 2(C), the CV curve of SA/CMCS/PPy hydrogel showed two oxidation peaks (196 and $529 \mathrm{mV}$ ) and two reduction peaks (344 and $-534 \mathrm{mV}$ ). The presence of these two pairs of reversible redox peaks confirmed that the SA/CMCS/PPy hydrogel possessed an excellent electrochemical activity. The conductivity of the hydrogel was measured by the 4-point probe method. It can be seen from the Table 1 that the conductivity of the SA/ CMCS/PPy hydrogel increased from $7.35 \times 10^{-6}$ to $8.03 \times$ $10^{-3} \mathrm{~S} \mathrm{~cm}^{-1}$ with the addition of polypyrrole. When the specific gravity of PPy was 0.2 , the conductivity of hydrogels was $1.42 \times 10^{-3} \mathrm{~S} \mathrm{~cm}^{-1}$. Conductivity of materials under this unit condition can respond well when subjected to electrical stimulation, and can produce effective regulation in cell culture..$^{22,35}$

A
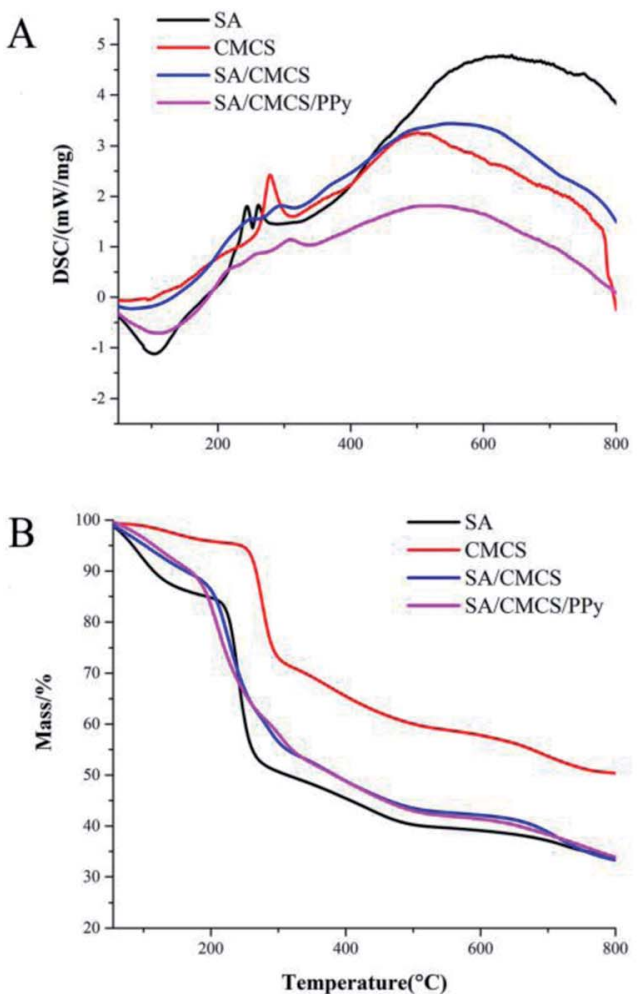

\subsection{Mechanical testing of the hydrogels}

According to the performance tests already done, the synthesis of hydrogels was suitable for the SA : CMCS's mass ratio of $2: 1$ and PPy's feed ratio of 0.2. The mechanical properties of the SA/ CMCS/PPy hydrogels were tested using a table universal testing machine (SHT4106, Mester Industrial System, China Ltd.). ${ }^{33,36}$ Tensile test results of the SA/CMCS/PPy hydrogels are shown in Fig. 2(D) and Table 2. The SA/CMCS hydrogel showed tensile stress (TS) of 0.07516 MPa and Young's modulus of 0.42952 MPa. After the addition of PPy, TS and Young's modulus slight decreased to $0.03823 \mathrm{MPa}$ and $0.17470 \mathrm{MPa}$. This may be due to the dominant influences of the inherent structural brittleness of PPy on the hydrogels and the support of the dispersed PPy particles on the structure of the whole hydrogel is limited. But with the increase of PPy content, the TS and Young's modulus of SA/CMCS/ PPy hydrogels showed a slow increase to a certain extent. The TS increased from $0.03823 \mathrm{MPa}$ to $0.06494 \mathrm{MPa}$, and Young's modulus increased from 0.17470 MPa to 0.40595 MPa. When the feed ratio of PPy was 0.2, the TS and Young's modulus of the SA/ CMCS/PPy hydrogel were 0.04902 and 0.28898 . The reason for this phenomenon is probably the positively charged PPy can interact with hydrogel basic skeleton by electrostatic interactions, of which molecular interactions could promote the mechanical and structural stability of the SA/CMCS/PPy hydrogels. ${ }^{8}$

\subsection{Effects of types on the thermal stability}

The thermal properties of the hydrogels were characterized by DSC and TGA (Fig. 3(A and B)). ${ }^{37}$ Fig. 3(A) shows that the DSC

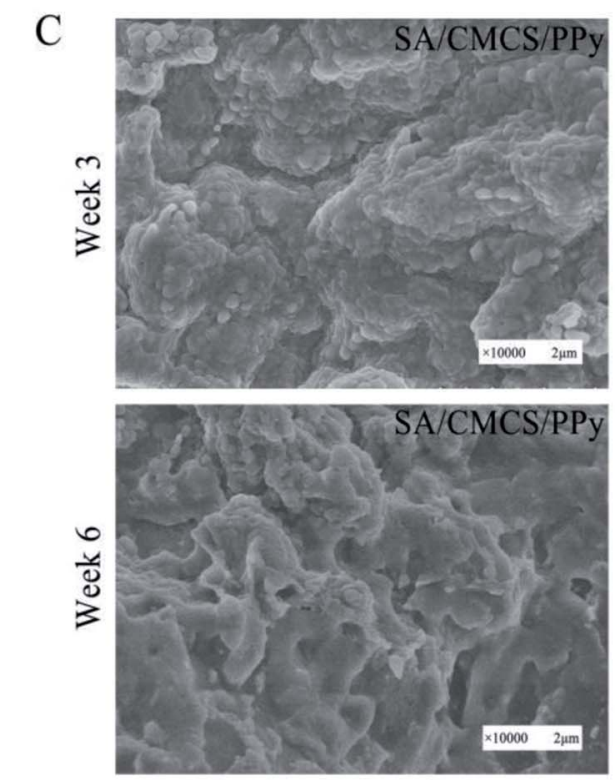

Fig. 3 (A) and (B) DSC and TGA curves of samples. (C) Scanning electron microscopy images of degradation of SA/CMCS and SA/CMCS/PPy hydrogel. 
curves of the SA, CMCS, SA/CMCS hydrogel and SA/CMCS/PPy hydrogel by the heating scan. The decomposition heat peak of SA from information analysis was $252.2{ }^{\circ} \mathrm{C}$, and the two decomposition heat peaks of CMCS were $278.3^{\circ} \mathrm{C}$ and $500.9{ }^{\circ} \mathrm{C}$. By using DSC, the behaviors of thermal kinetics and thermal decomposition of the hydrogels were observed. The SA/CMCS hydrogel had two decomposition heat peaks at $259.7{ }^{\circ} \mathrm{C}$ and $314.5^{\circ} \mathrm{C}$. It indicates that the hydrogel contains unreacted long chain segments of polymer monomers CMCS and SA, and the SA/CMCS polymer which is crosslinked by ion, is also included. When PPy was added to the SA/CMCS hydrogel, two obvious decomposition heat peaks were observed at $336.5{ }^{\circ} \mathrm{C}$ and $533.5^{\circ} \mathrm{C}$, and at the same time there was a peak at $108.6{ }^{\circ} \mathrm{C}$. The peak $108.6{ }^{\circ} \mathrm{C}$ was considered to be the result of the thermal decomposition of small molecules and de doping of PPy. Then, with the increase of temperature, the polymer molecules of long chain segments gradually decomposed. In addition, the thermal stability of the hydrogels was investigated by TGA under $\mathrm{N}_{2}$ atmosphere (Fig. 3(B)). The temperatures of the $240.9^{\circ} \mathrm{C}$ and $275.7^{\circ} \mathrm{C}$ were the fastest time of the weight loss of SA and CMCS, respectively. By observing the TGA curve of the SA/CMCS hydrogel, it can be seen that the mass slowly decreases with the increase of temperature, and the same situation happens in the TGA analysis of SA/CMCS/PPy hydrogel. It coincides with the result of DSC analysis. With the crosslinking of SA and CMCS and PPy doping, the chemical structure of hydrogels had changed, and the thermodynamic properties had changed accordingly.

A
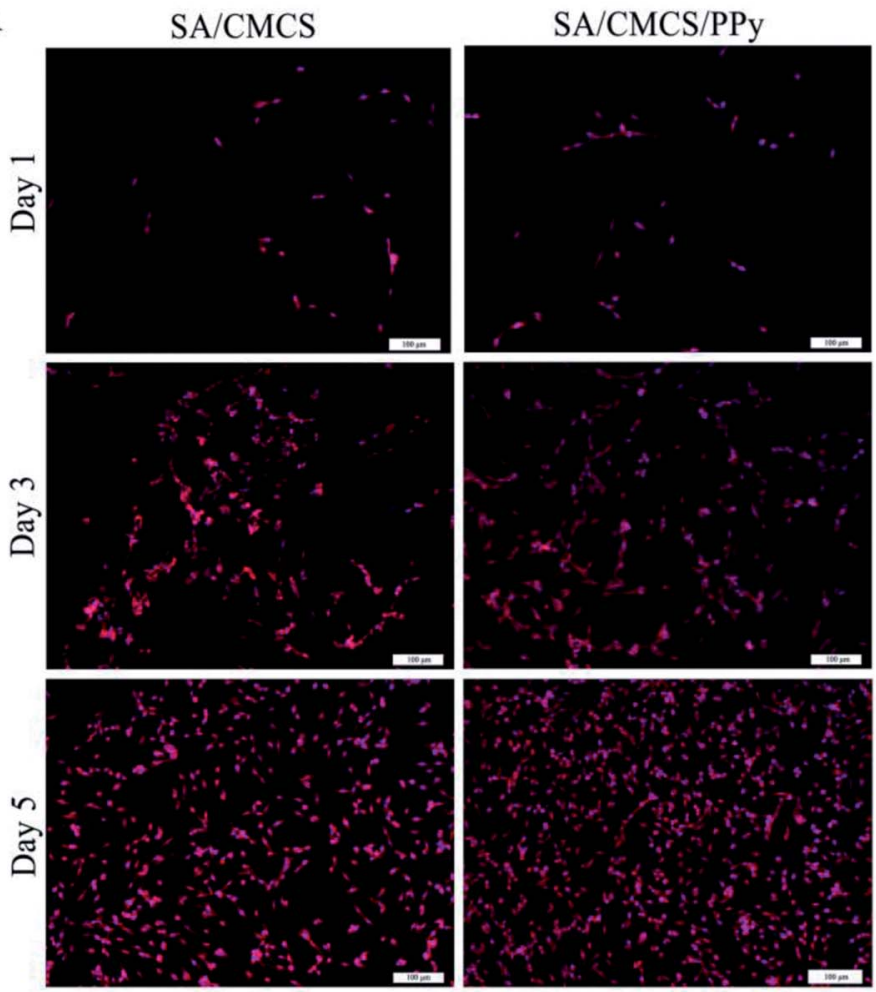

\subsection{Degradation of the SA/CMCS/PPy hydrogel}

The biodegradation behavior of the SA/CMCS/PPy hydrogel was evaluated by soaking the samples in PBS for 3 and 6 weeks at $37{ }^{\circ} \mathrm{C}^{38}$ After 3 weeks of soaking, the SA/CMCS/PPy hydrogel showed no significant morphological changes. At the same time, it was observed that the SA/CMCS hydrogel network skeleton was degraded to a certain extent, and some of the PPy particles attached to the interior of the hydrogel were exposed (Fig. 3(C)). After 6 weeks of degradation, it could be observed that some parts of the whole hydrogel exhibited obvious collapse and fracture, and the PPy particles attached to the surface of the hydrogel also showed shedding phenomenon. The result confirms the biodegradability of the SA/CMCS/PPy hydrogel under in vitro conditions. On the other hand, in a short period, the hydrogel has the ability to support its role as a tissue engineering material or an internal filling material for nerve conduit in a certain period.

\subsection{In vitro cell study}

PC12, RSC96 and BMMSC cells were cultured on the hydrogels to study the feasibility of their use as biological tissue materials particularly for neural applications. After condition screening, the SA/CMCS/PPy conductive hydrogel that SA : CMCS' mass ratio of $2: 1$ and PPy's feed ratio of 0.2 was selected as the experimental material. All the hydrogels were made into a regular smooth wafer after reaching the swelling balance and were placed in the 24-well plate for cell culture. The cells were inoculated on the surface of the material for co culture, and the suitable culture medium were used to cultivate different cells. ${ }^{39,40}$ As shown in Fig. 4(A), the

B
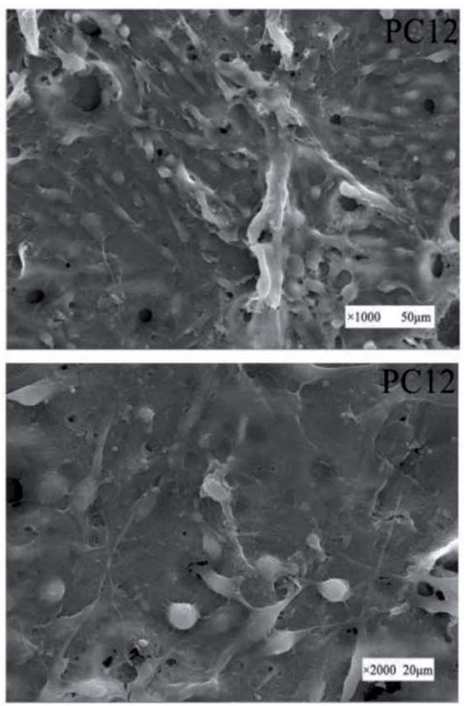

Fig. 4 (A) Representative fluorescence images of PC12 cells on SA/CMCS and SA/CMCS/PPy hydrogels cultured for 1, 3, and 5 days. (B) Scanning electron microscopy images of growth and adhesion of PC12 cells on SA/CMCS/PPy hydrogel. 

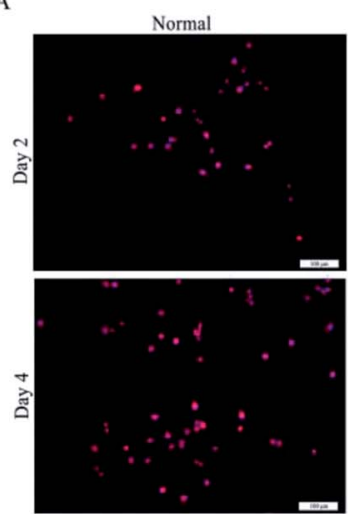
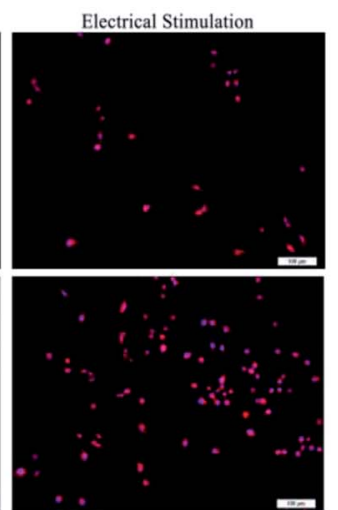

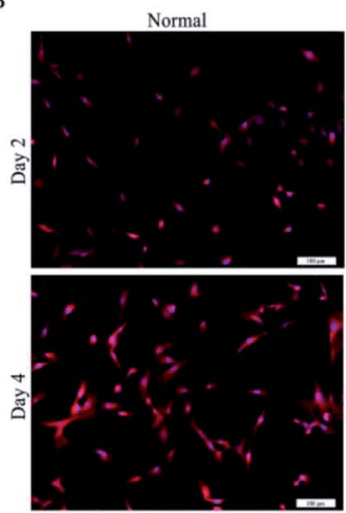

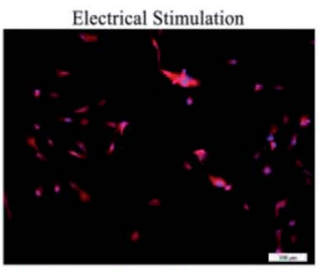

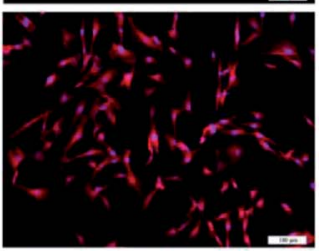

Fig. 5 (A) and (B) Representative fluorescence images RSC96 and BMMSC cells on SA/CMCS/PPy hydrogels cultured for 2 and 4 days, and the electrical stimulation cell group stimulated with $100 \mathrm{mV}$ for 2 hours each 24 hours.

number of living PC12 cells on both of the SA/CMCS and SA/ CMCS/PPy hydrogels increased with culture periods. By observing the distribution of the cells on the surface of the hydrogels, it could be seen that the distribution of SA/CMCS/PPy hydrogel was more uniform than SA/CMCS hydrogel. It is presumed that there are many holes in the SA/CMCS hydrogel scaffold, and the depth of a part of the hole is larger, which affects the growth and proliferation of the cells. When PPy was added to hydrogel, PPy particles adhered to hydrogel scaffolds, and filled some holes as supports, which provided favorable conditions for cell adhesion and proliferation. By conducting SEM to the hydrogels, the cells were observed to grow well and multiple protrusions were adhered to the surface of the SA/CMCS/PPy hydrogel (Fig. 4(B)). PC12 cells on hydrogels showed the high cell proliferation, indicating the good biocompatibility of SA/CMCS/PPy hydrogel. The RSC96 and BMMSC cells were inoculated on the surface of the material for co culture, and electrical stimulation was applied with $100 \mathrm{mV}$ for 2 hours each 24 hours. ${ }^{\text {20,41,42 }}$ After the cell inoculation, the regular electrical stimulation was carried out every day from the third day. The effects of electrical stimulation on proliferation and differentiation of RSC96 cells and BMMSC cells were observed respectively on second day and forth day (Fig. 5(A and B)). RSC96 cells can grow and proliferate smoothly on the hydrogels. After electrical stimulation, some cells developed axons, and the shape changed from the original circular growth to long shuttle type. The BMMSC cells were observed in the same way. Compared with the two sets of BMMSC cells after electric stimulation, it was found that the cells cultured on SA/CMCS/PPy hydrogel grew well, and showed plate-like adhesion on the surface, indicating that the cells were spreading better. In order to give the hydrogels a good electrical conductivity, PPy was added into the hydrogels. The SA/CMCS/PPy conductive hydrogels are expected to provide good material exchange and metabolic sites for cells, and can promote cell growth and increment under certain electrical stimulation conditions. All these results show that the SA/CMCS/PPy hydrogels have good biocompatibility and can provide strong support for cell growth and proliferation as tissue biomaterials.
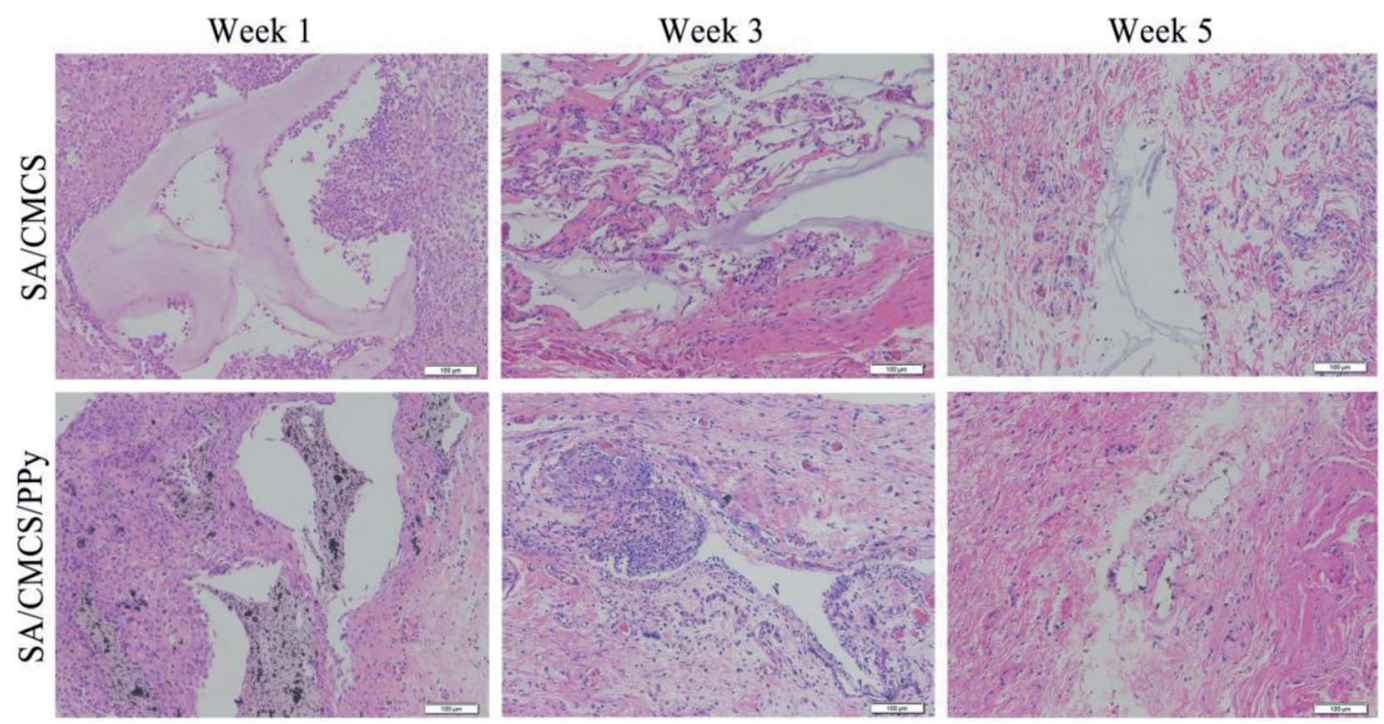

Fig. 6 Foreign body response by subcutaneous implantation of SA/CMCS and SA/CMCS/PPy hydrogels in Sprague Dawley rats (scale bar: 100 $\mathrm{mm})$. Implants and surrounding tissues were harvested after 1, 3 and 5 weeks implantation for H\&E staining. 
A
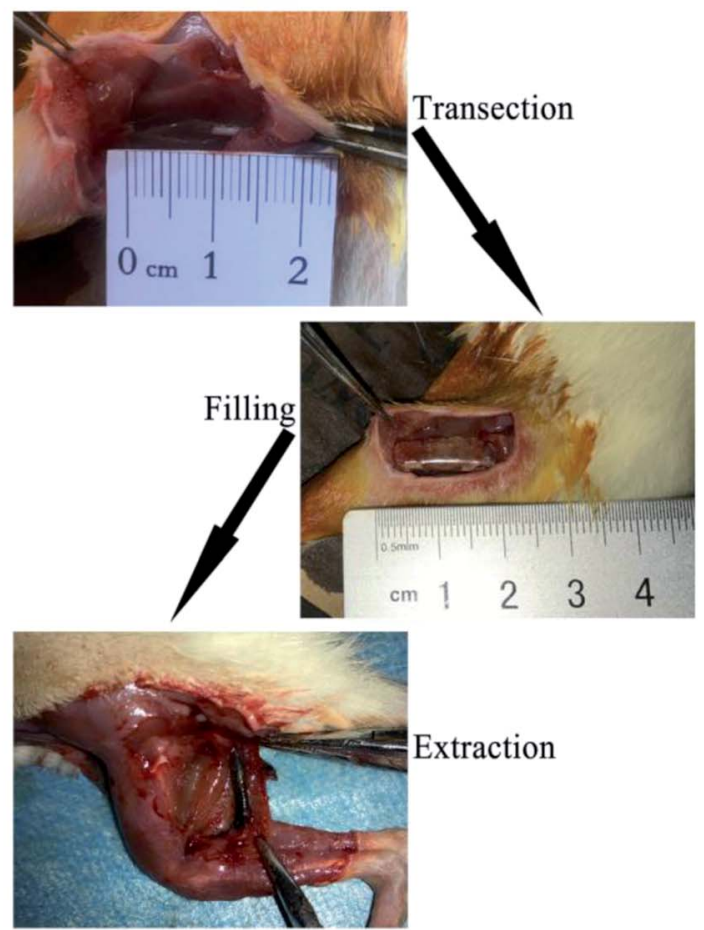

B
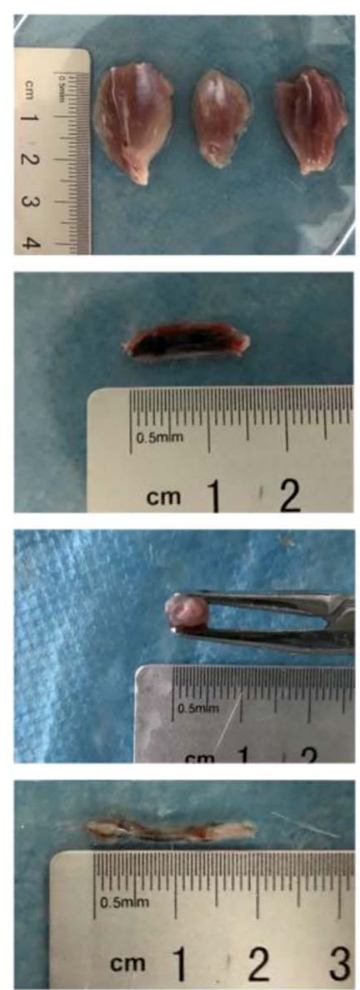

Fig. 7 (A) The schematic summary of the implantation and extraction surgery process. (B) The complete resection of the gastrocnemius, the nerve wrapped by the nerve conduit and hydrogel, and the regenerated sciatic nerve.

\subsection{In vivo inflammatory response after subcutaneous injection}

The biocompatibility of the conductive hydrogels was also studied in vivo. ${ }^{43,44}$ Two groups of Sprague-Dawley rats were subcutaneously implanted with SA/CMCS hydrogel and SA/ CMCS/PPy hydrogel. Foreign body response was studied by subcutaneous implantation in SD rats as shown in Fig. (6), H\&E staining was used to observe inflammatory conditions. Inflammatory cells, such as neutrophils, lymphocytes, macrophages, and other inflammatory cells can be distinctly distinguished from normal tissues. Inflammatory cells were recruited to the vicinity of the hydrogel at week 1 , indicating basal inflammatory responses to hydrogels, and the integrity of the hydrogel tissues can be observed. The number of these cells was decreased after 3 weeks, but there were still aggregated inflammatory cells around the SA/CMCS/PPy hydrogel. Probably because the hydrogel began to degradation, the PPy particles in the hydrogel were exposed and infiltrated into the adjacent tissue. After 5 weeks, no obvious inflammatory reaction was observed in the two kinds of hydrogels. Some of the remaining hydrogels were gradually entangled with the surrounding skin and muscle tissue. Severe inflammatory tissue responses to the hydrogels were not observed in all of the samples, and the good biocompatibility of the conductive hydrogel was demonstrated by the subcutaneous inflammatory reaction.
A

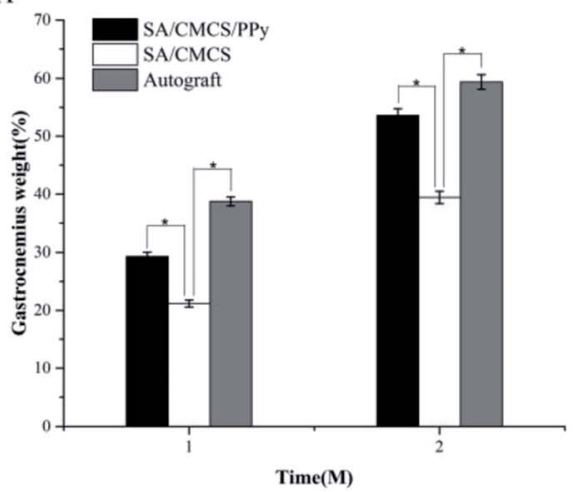

B

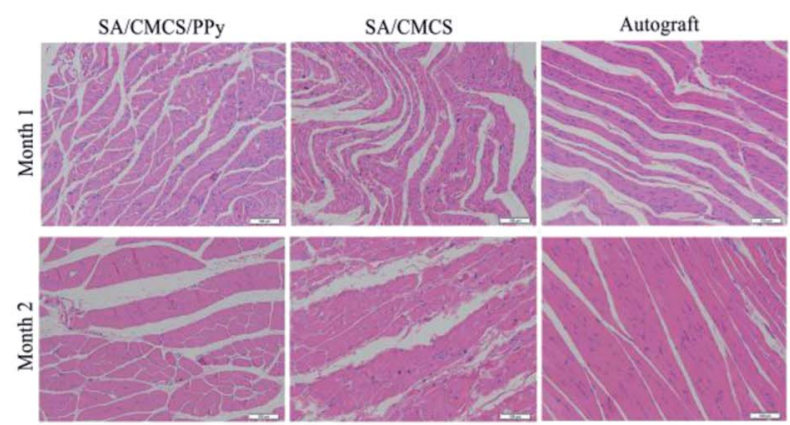

Fig. 8 (A) Recovery rate of gastrocnemius weight $(n=6, * p<0.05)$. (B) H\&E staining of the gastrocnemius of the experimental side. 


\subsection{Animal experiment of nerve conduit filling material}

In order to verify that the conductive hydrogel had the potential to be a practical biomaterial, the SA/CMCS/PPy hydrogel was tested as a nerve conduit filling material in rat experiment. ${ }^{\mathbf{4 5 - 4 7}}$ According to the experimental steps mentioned in the method, Fig. 7(A and B) shows the complete process of operation and extraction. After the experiment, the target muscles and nerves were evaluated. The tissue materials were removed at 1 and 2 months, and the target gastrocnemius was completely removed from the experimental side and the normal side of the calf. After removing the attached blood on the surface of the retrieved tissue, the wet weight of gastrocnemius was weighed by precision electronic balance. Comparison of the weight of the gastrocnemius muscles on both sides, gastrocnemius weight $(\%)=$ (gastrocnemius weight of the operated side)/ (gastrocnemius weight of the normal side) $\times 100 \%$. The recovery rates of the gastrocnemius weight in each experiment group at 1 and 2 months were shown in Fig. 8(A). From 1 month to 2 months, the recovery rates of the gastrocnemius weight all increased steadily. It was obvious that the recovery effects of the autologous nerve graft and the SA/CMCS/PPy hydrogel were better than the SA/CMCS hydrogel. The predominance of autograft in the early stage was prominent, but the recovery effect of the SA/CMCS/PPy hydrogel group was excellent at two months and was close to the autograft group. After that, the H\&E staining of the gastrocnemius muscles of the operated side was analyzed (Fig. 8(B)). At the time of the 1 month, the muscles were atrophied to varying degrees and there were obvious inflammatory reactions. At second months, the muscle atrophy of each group decreased significantly and the inflammatory

A
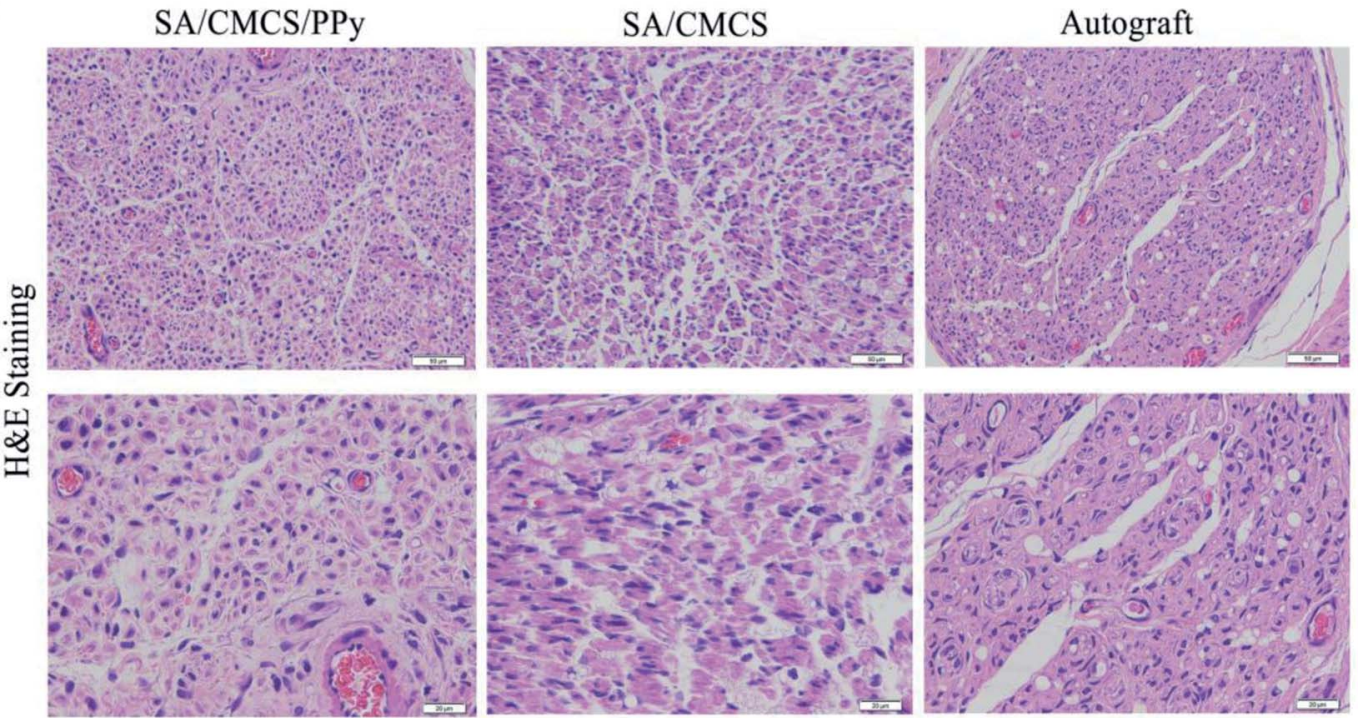

B
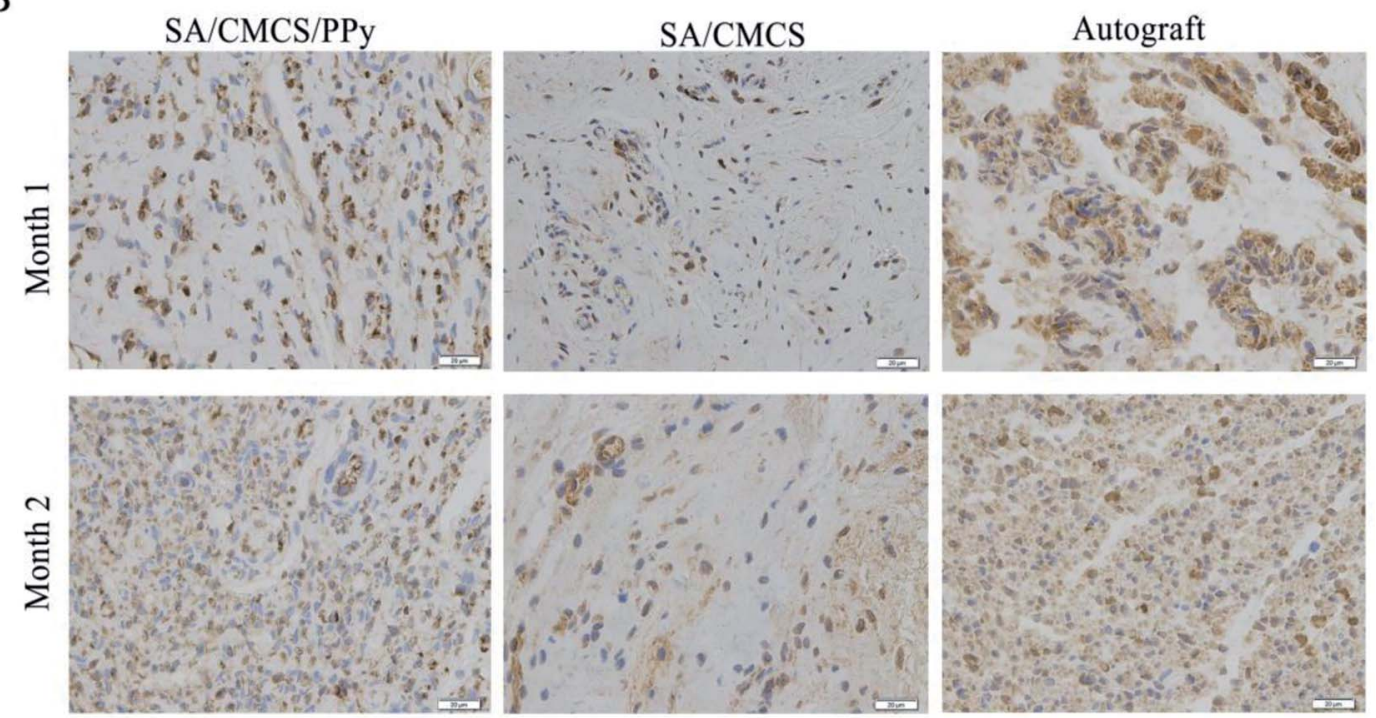

Fig. 9 (A) H\&E staining results of the regenerated nerve 2 months after surgery, scale bar: $50 \mu \mathrm{m}$ and $20 \mu \mathrm{m}$. (B) Immunohistochemical staining of the S-100 protein of the regenerated nerve after 1 and 2 months. 
reaction was reduced, it can be observed that the atrophy of the autograft group is mild. As time goes on, the muscle of SA/ CMCS/PPy hydrogel group became stronger and the diameter of muscle fibers increased significantly, while the SA/CMCS hydrogel group showed a general performance. The results of recovery rate of the gastrocnemius weight and $\mathrm{H} \& \mathrm{E}$ staining were consistent. The SA/CMCS/PPy hydrogel can promote the control of the regenerated nerve to the target muscles, relieve muscle atrophy and prevent denaturation.

H\&E staining was performed to observe the regenerated nerve morphology. As shown in Fig. 9(A), lots of injured nerve bundles with irregular or incomplete ring shape were observed in all groups. The number of new nerve fibers in the SA/CMCS hydrogel group was less and the tangent arrangement was relatively disordered. The improvements were more significant by SA/CMCS/PPy hydrogel and autograft groups, which resulted in the better level of morphological restoration and regularity of nerve bundles. The development of the regenerated nerve of the SA/CMCS/PPy hydrogel group was excellent, and the density and morphology of the nerve fibers were regular and even better than the autologous nerve group. S-100 protein is associated with the functions of the response and proliferation of Schwann cells, the brownish positive expression of S-100 protein indicates mature Schwann cells and regenerated nerves (Fig. 9(B)). At month 1, the positive expression of S-100 in SA/CMCS hydrogel group was sparse. The positive expression of SA/ CMCS/PPy hydrogel group was uniform but the expression in autograft group was more intensive. After two months, the distribution of SA/CMCS hydrogel group was irregular, and the regenerated nerve morphologies of SA/CMCS/PPy hydrogel and autograft groups were more mature and complete. The results of S-100 immunohistochemical experiment indicated that the function recovery of the regenerated nerve of the SA/CMCS/PPy hydrogel and autograft groups were better than that of SA/CMCS hydrogel. All the results suggest that the hydrogel can be used as a filling material for the nerve conduit and the potential of acting as a functional biological material.

\section{Conclusion}

Conductive SA/CMCS/PPy hydrogel with good mechanical and biocompatibility properties was successfully prepared. The hydrogel was obtained by crosslinking sodium alginate (SA) and carboxymethyl chitosan (CMCS) with calcium ions $\left(\mathrm{Ca}^{2+}\right)$, the doped polypyrrole (PPy) particles provide electrical conductivity. When the mass ratio SA : CMCS is $2: 1$ and the feed ratio of PPy is 0.20 , the mechanical performance of the conductive hydrogel is good. The conductivity can adjust by the PPy content and the suitable conductivity is beneficial to cell growth. The SA/CMCS/ PPy conductive hydrogel exhibited high biocompatibility in the culture of PC12, RSC96 and BMMSC cells, and its extracellular matrix (ECM)-mimicked structure provided good conditions for cell adhesion and proliferation. The in vivo biocompatibility of the conductive hydrogel was confirmed by subcutaneous inflammatory reaction, and the conductive hydrogel could play a full role as a filling material in the nerve conduit to provide strong support for peripheral nerve regeneration. All these results indicated the conductive hydrogel is an excellent candidate for biomedical applications.

\section{Conflicts of interest}

There are no conflicts to declare.

\section{Acknowledgements}

The financial support from the National Natural Science Foundation of China (No. 51473130, No. 51572206, 51403168), Wuhan Huanghe Excellence Plan, the National Key Research and Development Program of China (2016YFC1101605) and Entrepreneurship Training Program of Wuhan University of Technology (No. 20171049720018, 20171049720019 and 20171049720009) are kindly acknowledged.

\section{Notes and references}

1 J. Goding, A. Gilmour, P. Martens, L. Poole-Warren and R. Green, Adv. Healthcare Mater., 2017, 6, DOI: 10.1002/ adfm.201702969.

2 T. Sherstova, B. T. Stokke, B. Skallerud, G. Maurstad and V. E. Prot, Soft Matter, 2016, 12, 7338-7349.

3 K. Wang, K. C. Nune and R. D. Misra, Acta Biomater., 2016, 36, 143-151.

4 D. Pasqui, P. Torricelli, M. De Cagna, M. Fini and R. Barbucci, J. Biomed. Mater. Res., Part A, 2014, 102, 15681579.

5 J. Yang and C. Han, ACS Appl. Mater. Interfaces, 2016, 8, 25621-25630.

6 J. Liu, F. Cheng, H. Grenman, S. Spoljaric, J. Seppala, E. E. J. Sudaxshina Murdan, S. Willfor and C. Xu, Carbohydr. Polym., 2016, 148, 259-271.

7 S. Murdan, J. Controlled Release, 2003, 92, 1-17.

8 S. Yang, L. Jang, S. Kim, J. Yang, K. Yang, S. W. Cho and J. Y. Lee, Macromol. Biosci., 2016, 16, 1653-1661.

9 J. Liuyun, L. Yubao and X. Chengdong, J. Biomed. Sci., 2009, 16, 65.

10 F. Wang, Y. Wen and T. Bai, Mater. Sci. Eng., C, 2016, 69, 268275.

11 Y. Zhuang, F. Yu, H. Chen, J. Zheng, J. Ma and J. Chen, J. Mater. Chem. A, 2016, 4, 10885-10892.

12 A. W. Chan and R. J. Neufeld, Biomaterials, 2009, 30, 61196129.

13 P. Chansai, A. Sirivat, S. Niamlang, D. Chotpattananont and K. Viravaidya-Pasuwat, Int. J. Pharm., 2009, 381, 25-33.

14 E. O. Johnson, A. B. Zoubos and P. N. Soucacos, Injury, 2005, 36(suppl. 4), S24-S29.

15 W. W. Campbell, Clin. Neurophysiol., 2008, 119, 1951-1965. 16 B. Mammadov, M. Sever, M. Gecer, F. Zor, S. Ozturk, H. Akgun, U. H. Ulas, Z. Orhan, M. O. Guler and A. B. Tekinay, RSC Adv., 2016, 6, 110535-110547.

17 P. G. di Summa, P. J. Kingham, C. C. Campisi, W. Raffoul and D. F. Kalbermatten, Neurosci. Lett., 2014, 572, 26-31.

18 W. Huang, R. Begum, T. Barber, V. Ibba, N. C. Tee, M. Hussain, M. Arastoo, Q. Yang, L. G. Robson, S. Lesage, 
T. Gheysens, N. J. Skaer, D. P. Knight and J. V. Priestley, Biomaterials, 2012, 33, 59-71.

19 H. Xu, Y. Yan and S. Li, Biomaterials, 2011, 32, 4506-4516.

20 H. Xu, J. M. Holzwarth, Y. Yan, P. Xu, H. Zheng, Y. Yin, S. Li and P. X. Ma, Biomaterials, 2014, 35, 225-235.

21 H. Liu, C. Wang, Q. Gao, J. Chen, B. Ren, X. Liu and Z. Tong, Int. J. Pharm., 2009, 376, 92-98.

22 B. Sun, T. Wu, J. Wang, D. Li, J. Wang, Q. Gao, M. A. Bhutto, H. El-Hamshary, S. S. Al-Deyab and X. Mo, J. Mater. Chem. B, 2016, 4, 6670-6679.

23 C. Zhou, B. Liu, Y. Huang, X. Zeng, H. You, J. Li and Y. Zhang, J. Biomed. Mater. Res., Part A, 2017, 105, 3077-3085.

24 B. M. Lloyd, R. D. Luginbuhl, M. J. Brenner, B. G. Rocque, T. H. Tung, T. M. Myckatyn, D. A. Hunter, S. E. Mackinnon and G. H. Borschel, Microsurgery, 2007, 27, 138-145.

25 E. A. Growney Kalaf, M. Pendyala, J. G. Bledsoe and S. A. Sell, J. Mech. Behav. Biomed. Mater., 2017, 72, 229-240.

26 Q. Yu, Y. Zheng, N. Yan, Y. Xie, K. Qiao and R. Jin, RSC Adv., 2015, 5, 106953-106958.

27 T. W. Chung, D. M. Lai, S. D. Chen and Y. I. Lin, J. Biomed. Mater. Res., Part A, 2014, 102, 315-323.

$28 \mathrm{~S}$. Wu, B. Duan, A. Lu, Y. Wang, Q. Ye and L. Zhang, Carbohydr. Polym., 2017, 174, 830-840.

29 J. Pelto, M. Bjorninen, A. Palli, E. Talvitie, J. Hyttinen, B. Mannerstrom, R. Suuronen Seppanen, M. Kellomaki, S. Miettinen and S. Haimi, Tissue Eng., Part A, 2013, 19, 882-892.

30 D. Dehghan Baniani, R. Bagheri and A. Solouk, Carbohydr. Polym., 2017, 174, 633-645.

31 C. Gao, M. Liu, J. Chen and X. Zhang, Polym. Degrad. Stab., 2009, 94, 1405-1410.

32 M. A. Iannuzzi, R. Reber, D. M. Lentz, J. Zhao, L. Ma and R. C. Hedden, Polymer, 2010, 51, 2049-2056.

33 L. Li, J. Ge, P. X. Ma and B. Guo, RSC Adv., 2015, 5, 9249092498.
34 A. Mihic, Z. Cui, J. Wu, G. Vlacic, Y. Miyagi, S. H. Li, S. Lu, H. W. Sung, R. D. Weisel and R. K. Li, Circulation, 2015, 132, 772-784.

35 X. Liu, A. L. Miller II, S. Park, B. E. Waletzki, Z. Zhou, A. Terzic and L. Lu, ACS Appl. Mater. Interfaces, 2017, 9, 14677-14690.

36 W. Zhao, Z. Han, L. Ma, S. Sun and C. Zhao, J. Mater. Chem. $B, 2016,4,8016-8024$.

37 B. Wang, X. Wu, J. Li, X. Hao, J. Lin, D. Cheng and Y. Lu, Polymers, 2016, 8, 110.

38 H. Tanuma, T. Saito, K. Nishikawa, T. Dong, K. Yazawa and Y. Inoue, Carbohydr. Polym., 2010, 80, 260-265.

39 K. Marycz, A. Śmieszek, J. Grzesiak, A. Donesz-Sikorska and J. Krzak-Roś, Biomed. Mater., 2013, 8, 065004.

40 W. Zhang, L. Zhang, J. Liu, L. Zhang, J. Zhang and P. Tang, Biomaterials, 2017, 142, 90-100.

41 Y. C. Lin, C. H. Kao, C. C. Chen, C. J. Ke, C. H. Yao and Y. S. Chen, PLoS One, 2015, 10, e0116711.

42 Z. Zhang, X. Li, S. Zuo, J. Xin and P. Zhang, Neural Regener. Res., 2014, 9, 1075-1078.

43 H. Y. Zhou, Y. P. Zhang, W. F. Zhang and X. G. Chen, Carbohydr. Polym., 2011, 83, 1643-1651.

44 J. Sun, G. Jiang, T. Qiu, Y. Wang, K. Zhang and F. Ding, J. Biomed. Mater. Res., Part A, 2010, 95, 1019-1027.

45 T. M. Hopkins, K. J. Little, J. J. Vennemeyer, J. L. Triozzi, M. K. Turgeon, A. M. Heilman, D. Minteer, K. Marra, D. B. Hom and S. K. Pixley, J. Biomed. Mater. Res., Part A, 2017, 105, 3148-3158.

46 K. A. Alberti, C. I. Neufeld, J. Wang and Q. Xu, ACS Biomater. Sci. Eng., 2016, 2, 937-945.

47 H. Xie, W. Yang, J. Chen, J. Zhang, X. Lu, X. Zhao, K. Huang, H. Li, P. Chang, Z. Wang and L. Wang, Adv. Healthcare Mater., 2015, 4, 2195-2205. 